\title{
Xylan hydrolysis in Populus trichocarpa $x$ P. deltoides and model substrates during hydrothermal pretreatment
}

Heather L. Trajano ${ }^{\mathrm{a}, \mathrm{b}, \mathrm{f}}$, Sivakumar Pattathil ${ }^{\mathrm{c}, \mathrm{f}}$, Bruce A. Tomkins ${ }^{\mathrm{d}, \mathrm{f}}$, Timothy J. Tschaplinski ${ }^{\mathrm{e}, \mathrm{f}}$, Michael G. Hahn ${ }^{\mathrm{c}, \mathrm{f}}$, Gary J. Van Berkel ${ }^{\mathrm{d}, \mathrm{f}}$, and Charles E. Wyman ${ }^{\mathrm{a}, \mathrm{f}^{*}}$

${ }^{\mathrm{a}}$ Department of Chemical and Environmental Engineering and Center for Environmental Research and Technology, Bourns College of Engineering, University of California Riverside, 1084 Columbia Ave, Riverside, CA, USA, 92507.

${ }^{\mathrm{b}}$ Permanent address: Department of Chemical and Biological Engineering, 2360 East Mall, Vancouver, British Columbia, Canada, V6T 1 Z3.

${ }^{\mathrm{c} C o m p l e x}$ Carbohydrate Research Center, The University of Georgia, 315 Riverbend Rd., Athens, GA, USA, 30602.

${ }^{\mathrm{d}}$ Chemical Sciences Division, Oak Ridge National Laboratory, PO Box 2008 MS6341, Oak Ridge, TN, USA, 37831.

${ }^{\mathrm{e}}$ Biosciences Division, Oak Ridge National Laboratory, PO Box 2008 MS6341, Oak

Ridge, TN, USA, 37831

${ }^{\mathrm{f}}$ BioEnergy Science Center, Oak Ridge National Laboratory, PO Box 2008 MS6341, Oak Ridge, TN, USA, 37831.

HLT: heather.trajano@ubc.ca

4M KOH PC: $4 \mathrm{M} \mathrm{KOH}$ extraction post(P) sodium chlorite(C) extraction; AG: arabinogalactan; AIR: alcohol insoluble residue; BX: birchwood xylan; DI: deionized; DP: degree of polymerization; ELISA: enzyme-linked immunosorbent assay; HC: holocellulose; HG: homogalacturonan backbone; HPLC: high pressure liquid chromatography; mAbs: monoclonal antibodies; m/z: mass to charge ratio; PtPd: Populus trichocarpa $x$ P. deltoides; RG-I/AG: pectic-arabinogalactan; UPLC-MS: ultra-high performance liquid chromatography-mass spectrometry; XG: xyloglucans 
SP: siva@ccrc.uga.edu

BAT: tomkinsba@ornl.gov

TJT: tschaplinstj@ornl.gov

MGH: hahn@ccrc.uga.edu

GJVB: vanberkelgj@ornl.gov

*CEW: cewyman@engr.ucr.edu, 951 781-5703

\begin{abstract}
Previous studies defined easy and difficult to hydrolyze fractions of hemicellulose that may result from bonds among cellulose, hemicellulose, and lignin. To understand how such bonds affect hydrolysis, Populus trichocarpa $x$ P. deltoides, holocellulose isolated from Populus trichocarpa $x$ P. deltoides and birchwood xylan were subjected to hydrothermal flow-through pretreatment. Samples were characterized by glycome profiling, HPLC, and UPLC-MS. Glycome profiling revealed steady fragmentation and removal of glycans from solids during hydrolysis. The extent of polysaccharide fragmentation, hydrolysis rate, and total xylose yield were lowest for Populus trichocarpa $x$ P. deltoides and greatest for birchwood xylan. Comparison of results from Populus trichocarpa $x$ P. deltoides and holocellulose suggested that lignin-carbohydrate complexes reduce hydrolysis rates and limit release of large xylooligomers. Smaller differences between results with holocellulose and birchwood xylan suggest xylan-cellulose hydrogen bonds limited hydrolysis, but to a lesser extent. These findings imply cell wall structure strongly influences hydrolysis.
\end{abstract}




\section{Keywords}

hydrolysis; hemicellulose; lignin-carbohydrate complex

\section{Introduction}

Growing demand for petroleum derived fuels coupled with the need to reduce greenhouse gas emissions have prompted the search for renewable fuels.

Lignocellulose is the only sufficiently abundant low cost resource for large-scale economic production of liquid transportation fuels. Biological conversion of lignocellulose to ethanol relies upon pretreatment to overcome the inherent recalcitrance of biomass to enzymatic hydrolysis. Some have concluded that hemicellulose removal is a key to increasing enzymatic digestibility (Kumar and Wyman, 2010; Leu and Zhu, 2013). Hydrothermal pretreatment with water at elevated temperatures removes hemicellulose through hydrolysis to oligomers and monomers (Kim et al., 2013). 
The hemicellulose of Populus trichocarpa $\mathrm{x}$ P. deltoides wood, a promising North American biofuel feedstock, consists primarily of xylans (Sannigrahi et al., 2010). Glucuronoxylans are the primary hemicellulose in the secondary cell walls of poplar wood and have a xylan backbone with $O$-acetyl substitutions and 4-O-methyl $\alpha-D$-glucosyluronic acid side chain residues (Albersheim et al., 2011). Cellulose and hemicelluloses are thought to interact via hydrogen bonds to form important polysaccharide networks within plant cell walls (Abramson et al., 2013; Faik, 2013). Hemicellulose is also covalently bound to lignin through ester linkages, ether linkages, and indirect ester-ether bridges to form lignin-carbohydrate complexes (Albersheim et al., 2011).

Kobayashi and Sakai (1956) were the first to note that the initial rate of xylan hydrolysis was much faster compared to the rate later in pretreatment; they attributed this to the presence of fast reacting and slow reacting xylan fractions. Multiple kinetic models have since incorporated this biphasic behavior (Conner, 1984; Garrote et al., 1999; Kim et al., 2013; Maloney and Chapman, 1985; Zhu et al., 2012). The most frequently proposed cause for this behaviour is differences in intrinsic reactivity due to cell wall structure (Maloney and Chapmen, 1985), lignincarbohydrate bonds (Chen et al., 2010; Conner, 1984; Lee et al., 1999) and hemicellulose-cellulose interactions (Lee et al., 1999). Although many have suggested such features could explain the biphasic breakdown of hemicellulose, limited results to date verify this hypothesis. Development of more mechanistic hydrolysis models requires a deeper understanding of how cell wall structure affects 
hemicellulose hydrolysis (Abatzoglou et al., 1992; Gray et al., 2007; Liu and Wyman, 2003).

The absence of such models is in part due to the challenge of characterizing biomass; sophisticated chemical analyses do not lend themselves to process development efforts. The recent growth in the number of monoclonal antibodies (mAbs) and probes such as carbohydrate binding modules now enable sophisticated and rapid studies of cell wall composition and structure (Knox, 1997; Pattathil et al., 2012; Willats et al., 2000).

A second challenge to the detailed analysis of hemicellulose hydrolysis is the use of batch reactors. Xylose and xylooligomers react to generate products such as furfural and pseudo-lignin (Kim et al., 2013; Saeman, 1945; Sannigrahi et al., 2011), and cooling results in product precipitation (Gray et al., 2007). In a fixed bed flowthrough reactor, soluble products are rapidly removed from the reactor after formation, thus improving the development of product profiles as a function of time (Liu and Wyman, 2003; Mok and Antal, 1992; Song et al., 2012). Pumping, heating, and treating large volumes of water for flow-through pretreatment would be difficult on a commercial scale; therefore flow-through reactors are currently limited to laboratory use, where they nonetheless provide useful information about the pretreatment process.

The objective of this study is to determine the influence of xylan-cellulose hydrogen bonds and xylan-lignin bonds on the hydrolysis of poplar xylan. $P$. trichocarpa $\mathrm{x}$ P. deltoides, holocellulose (i.e., the cellulose and hemicellulose fraction left after delignification of the cell wall) isolated from P. trichocarpa $\mathrm{x} P$. 
deltoides, and birchwood xylan were pretreated in a fixed bed flow-through reactor.

Differences in glycome profiles of the pretreated solids, xylan removal, and

oligomer release from $P$. trichocarpa $\mathrm{x} P$. deltoides and holocellulose provided insights into the influence of carbohydrate-lignin bonds on xylan removal, while differences in the behaviour of holocellulose and birchwood xylan reflected the influence of xylan-cellulose hydrogen bonds.

\section{Materials and Methods}

\subsection{Populus trichocarpa x P. deltoides, holocellulose, and birchwood xylan}

Stemwood of hybrid cottonwood poplar Populus trichocarpa $x$ P. deltoides ‘53-239’ §’ was provided by Oak Ridge National Laboratory, TN. Logs were debarked, split with an axe, chipped (Yard Machines 10HP, MTD Products Inc., Cleveland, $\mathrm{OH}$ ), and knife milled (Model 4 Wiley Mill, Thomas Scientific, Swedesboro, NJ) through a $1 \mathrm{~mm}$ screen size. The material was further milled to particles with dimensions of $0.18 \mathrm{~mm}$ to $0.85 \mathrm{~mm}$ (Thomas-Wiley Laboratory Mill Model 4, Arthur H. Thomas Company, Philadelphia, PA).

Cellulose and hemicellulose were isolated from this material to produce holocellulose by first removing extractives following the procedure by Sluiter et al. (2005). Lignin was then removed from the extractive-free solids by the oxidative treatment outlined by Zhang et al. (2006) and Wickholm et al (1998). In brief, extractive-free milled $P$. trichocarpa $x$ P. deltoides stemwood was dispersed in deionized (DI) water (100mL/g sample) with sodium chlorite ( $40 \%$ by dry weight of sample) and glacial acetic acid (10\% by dry weight of sample) in a sealed plastic 
pouch (Kapak Corporation, Minneapolis, MN). The pouch was then placed in a reciprocating water bath at $70^{\circ} \mathrm{C}$ for one hour. This treatment was repeated three more times. The holocellulose was recovered by filtration and washed thoroughly with DI water. The second model substrate, birchwood xylan, was purchased from Sigma-Aldrich (Lot 010M0169). The composition of the three substrates is reported in Table 1.

\subsection{Pretreatment}

P. trichocarpa $x$ P. deltoides and model substrates were subjected to flowthrough pretreatment at $180^{\circ} \mathrm{C}$ using the flow-through reactor system and methods described by Trajano et al. (2013) with adjustments to the mass of biomass loaded, set pressure, and sand bath and reactor temperatures. Briefly, the primary system components are a pump, heating coil, reactor, and cooling coil. The heating coil and reactor are submerged in a fluidized sand bath, while the cooling coil is submerged in a water bath. Water is heated as it is pumped to the reactor. The product stream exits the reactor and is immediately cooled prior to collection; the product stream is not recycled to the reactor inlet. The reactor was loaded with $1 \mathrm{~g}$ biomass on a moisture free basis. A back pressure valve was adjusted to $1.1 \mathrm{MPa}$ gauge and water flow rate was adjusted to $20 \mathrm{~mL} / \mathrm{min}$. The sand bath was heated to $190^{\circ} \mathrm{C}$. Time zero for the reaction was defined as the point when the reactor temperature reached $178^{\circ} \mathrm{C}$. Reactions lasted 3 to 12 minutes; liquid samples were collected over successive 3 minute intervals. The reactor temperature fluctuated between $178^{\circ} \mathrm{C}$ and $181^{\circ} \mathrm{C}$ during each run with an average reactor temperature of $\sim 180^{\circ} \mathrm{C}$. A run 
was also performed in which the reactor was cooled immediately after reaching $178^{\circ} \mathrm{C}$; this generated the material designated as $0 \mathrm{~min}$.

\subsection{Glycome profiling}

Glycome profiling (Pattathil et al., 2012) consists of enzyme-linked immunosorbent assay (ELISA) screening of solubilized cell wall components recovered from solid substrates by sequential extractions of increasing severity against a comprehensive toolkit of cell wall glycan-directed monoclonal antibodies (Pattathil et al., 2010) to examine cell wall compositional and structural changes caused by hydrothermal pretreatment by identifying the glycan components present in each extract. The most loosely bound components are solubilized by the first extraction (oxalate), whereas the most tightly bound components are released in the last (4M KOH post chlorite extraction- 4M KOH PC). Sequential cell wall extractions and ELISAs of biomass residues were performed as described by Pattathil et al. (2012). The ELISA plate was loaded with a constant amount of carbohydrate. The profiling was conducted as two technical replicates, and the data reported are the averages of the replicates. Plant cell wall glycan directed monoclonal antibodies were obtained as laboratory stocks of hybridoma cell culture supernatants from the Complex Carbohydrate Research Center; these antibodies can be obtained from CarboSource Services (www.carbosource.net: JIM, MAC and CCRC series). Other antibodies used were purchased from Biosupplies Australia (Parkville, Victoria, Australia, LAMP and BG-1 antibodies) and were used per the manufacturer's instructions. 


\subsection{Analysis of total sugar oligomer content of hydrolysates}

The post-hydrolysis procedure described by Sluiter et al. (2006) was used to determine the total sugar content of the hydrolysate. Sugars were detected by high pressure liquid chromatography (HPLC) using an Aminex HPX-87H column (BioRad, Hercules, CA) heated to $65^{\circ} \mathrm{C}$ with a separation module (Alliance 2695, Waters Associates, Milford, MA) equipped with a refractive index detector (Model 2414, Waters Associates, Milford, MA). The eluent was $0.005 \mathrm{M}$ sulphuric acid in the isocratic mode.

\subsection{Analysis of oligomer content $(D P \leq 6)$ of hydrolysates by ultra-high performance liquid chromatographic separations with mass spectrometric detection}

The hydrolysates were analyzed using ultra-high performance liquid chromatography with mass spectrometry (UPLC-MS) to identify and quantify xylooligomers with a degree of polymerization (DP) up to and including six. Standards, with purity >95\%, were purchased from Sigma-Aldrich (St. Louis, MO), Toronto Research Chemicals, Inc. (North York, Ontario, Canada) or Megazyme International Ireland (Bray, County Wicklow, Ireland), and were used as received. Standards were prepared in 80/20 (v/v) acetonitrile/water using Fisher Optima ${ }^{\circledR}$ LC/MS grade solvents (Fisher Scientific, Fair Lawn, NJ). The acetonitrile and water eluents were both prepared using Optima ${ }^{\circledR}$ grade solvent and contained $10 \mu \mathrm{M}$ reagent-grade sodium acetate as a modifier. 
All samples were filtered through a $0.2 \mu \mathrm{m}$ porosity nylon filter (Cat. 24137, Grace Davison Discovery Sciences, Deerfield, IL) prior to analysis. Each sample was diluted fifty- to five-hundred-fold using 80/20 (v/v) acetonitrile/water.

Oligomers were separated using an ACQUITYTM Ultra Performance Liquid Chromatograph equipped with a sample manager, binary solvent manager, and a BEH® HILIC UPLC column $(2.1 \mathrm{~mm}$ i.d. $\mathrm{x} 100 \mathrm{~mm}$ length, packed with $1.7 \mu \mathrm{m}$ diameter particles), all from Waters Associates (Milford, MA). The injection volume was $0.5 \mu \mathrm{L}$. The column oven and sample manager temperatures were maintained at 35 and $4^{\circ} \mathrm{C}$, respectively. A linear gradient separation employing acetonitrile/10 $\mu \mathrm{M}$ sodium acetate (Solvent $\mathrm{A}$ ) and water/10 $\mu \mathrm{M}$ sodium acetate (Solvent B), described in Table 2, was used. The eluent flow rate was maintained at $0.5 \mathrm{~mL} / \mathrm{min}$. Sample-to-sample analysis time was approximately $6 \mathrm{~min}$. All samples were analyzed in at least triplicate.

Sodiated analogs $\left(\mathrm{M}+\mathrm{Na}^{+}\right)$of the oligomers were formed in the ion source using micromolar concentrations of sodium acetate, which was added to the eluent. The resulting products were detected using an AB/SCIEX 4000 QTrap(r) System (AB/Sciex, Foster City, CA, USA) operated in the single-ion monitoring mode employing electrospray ionization in the positive ionization mode. Xylodextrins were detected at $m / z$ 305.1, 437.2, 569.2, 701.3, and 833.3, corresponding to xylobiose (DP2), -triose (DP3), -tetraose (DP3), -pentaose (DP5), and -hexaose (DP6), respectively. The instrument was optimized for detection of each target $\mathrm{m} / \mathrm{z}$ value. 


\subsection{Structural carbohydrate and Klason lignin analysis}

The compositions of the raw and pretreated solids were determined using the two-step acid hydrolysis procedure outlined by Sluiter et al (2011). Sugars were detected using the HPLC configuration described in Section 2.4.

\section{Results and discussion}

\subsection{Changes in glycan composition}

Cell wall compositional and structural changes caused by hydrothermal pretreatment were examined by glycome profiling; this was complemented by bulk compositional analysis. The glycome profiles of raw and pretreated $P$. trichocarpax P. deltoides are shown in Figure 1. Each column represents an increase in pretreatment time, and within each column, the series of increasingly severe extracts (as labelled at the bottom of each heat map) are depicted. The amount of material recovered in each extract was plotted in the top-most bar graphs. Thus, the relative abundance of an epitope is a function of both the amount of material recovered in each extract and the antibody binding intensity. Comparison of the amount of material recovered after each extraction step per gram of alcohol insoluble residue (AIR, top most bar graphs) from untreated material and material subjected to just heat up for pretreatment $(0 \mathrm{~min})$ showed that the heating period notably increased cell wall extractability, indicating a loosening of the cell wall. This cell wall loosening was also observed in the biomass recovered after 3 min of pretreatment. In addition, it can be seen that as soon as biomass was exposed to elevated temperature (0 min sample), significant amounts of arabinogalactan $(A G)$, pectic- 
arabinogalactan (RG-I/AG), and homogalacturonan (HG backbone) epitopes were no longer detectable in the mild oxalate and carbonate extracts. The drop in binding intensities of mAbs may be due to either removal of epitope structures from the cell wall glycans present in the residual solids or pretreatment-induced fragmentation of these polysaccharides to smaller molecules that cannot stick to the ELISA plate.

DeMartini et al. (2011) applied glycome profiling, molecular sieve chromatography, and immunolabeling to $P$. trichocarpa $\times P$. deltoides samples after batch hydrothermal pretreatment and found that the drop in binding intensity was due to both fragmentation of polysaccharides and epitope removal. As the biomass used in this study came from a tree in the same stand as the one used by DeMartini et al. (2011), the drop in binding intensity after flow-through pretreatment is likely due to similar changes. Epitopes recognized in $4 \mathrm{M} \mathrm{KOH} \mathrm{PC} \mathrm{extracts} \mathrm{of} \mathrm{the} \mathrm{untreated} \mathrm{and} 0$ min residues, were associated with lignin and not detected in the residues after $3 \mathrm{~min}$ of pretreatment, indicating removal and/or significant epitope fragmentation. Xylan epitopes, recognized by xylan-4 through xylan- 7 groups of mAbs and present in chlorite extracts from untreated and 0 min residues, were completely absent in residues subjected to $3,6,9$, and 12 min pretreatments. A progressive reduction in the abundance of xylan epitopes was observed in all non-chlorite extracts from residues subjected to $3,6,9$, and 12 min pretreatments. If one then also factors in the overall reduction in the amount of material recovered in all non-chlorite extraction steps among the 0 through 12 min pretreated samples, it is clear that the amount and size of xylan epitopes decreased with increasing pretreatment duration, although complete removal of xylan epitopes was not obtained even after $12 \mathrm{~min}$ of 
pretreatment. In a similar trend, increasing the pretreatment time also led to progressive removal and fragmentation of xyloglucan $(\mathrm{XG})$ epitopes. Thus, in general, the glycome profiles (Figure 1) show a drop in overall mAb binding intensities as pretreatment time increased, indicating steady removal and fragmentation of non-cellulosic glycans.

Glycome profiles of holocellulose before and after pretreatment are shown in Figure 2. No Klason lignin was detected in the holocellulose, as reported in Table 1. This observation is consistent with previously published compositional analyses (Ahlgren and Goring, 1971; Kumar et al., 2013). However, comparison of Figure 2 to Figure 1 provides further insight into the glycan content of holocellulose. From the first column of Figure 2, it is clear that the extractions and oxidative treatments used to prepare holocellulose leave the XG, xylan, glucan, HG, RG-I/AG, and AG epitopes intact, essentially resulting in a delignified cell wall. Epitope detection in the chlorite and 4M KOH PC extracts indicated that there were still lignin-xylan complexes in the holocellulose, but at a much lower level as evidenced by reduced recovery of material from these extractions (top most bar graph of Figure 2). Relative to untreated $P$. trichocarpa $x P$. deltoides, significantly more material was recovered from the mild oxalate and carbonate extractions of untreated holocellulose, but far less material was recovered from the chlorite and 4M KOH PC extractions of untreated holocellulose (top most bar graphs of Figure 2), indicating that oxidative delignification leads to a loosening of the cell wall. The overall binding of xylan antibodies (groups 5 to 7) decreased slightly in untreated 
holocellulose relative to untreated $P$. trichocarpa $x$ P. deltoides, suggesting partial fragmentation and removal of these epitopes during oxidative delignification.

It has been previously posited that lignin-carbohydrate complexes significantly affect the release of xylooligomers (Chen et al., 2010; Conner, 1984; Lee et al., 1999). Differences between the glycome profiles of pretreated holocellulose and those for $P$. trichocarpa $x P$. deltoides reflect this influence, since such bonds are significantly reduced in holocellulose. Very little material was extracted from pretreated holocellulose, even after the heating period, which indicates that the residual holocellulose is primarily cellulose with only a small amount of noncellulosic glycans. In addition, the overall signal strength in the ELISAs was much lower in holocellulose compared with $P$. trichocarpa $x P$. deltoides, even though each well was loaded with an equal amount of carbohydrate. As described above, DeMartini et al. (2011) found that such a reduction in signal strength correlated with loss and fragmentation of non-cellulosic glycans. The differences in mAbs binding intensity suggest that the removal and fragmentation of non-cellulosic glycans from holocellulose occurred to a greater extent than from $P$. trichocarpa $x P$. deltoides. Finally, Figure 2 shows that the drop in binding intensities of all glycan directed mAbs occurred more quickly in holocellulose with increasing pretreatment time than in $P$. trichocarpa $\times$ P. deltoides, indicating faster removal of glycans. For example, many of the non-fucosylated XG and xylans that remained in P. trichocarpa $x P$. deltoides, even after 12 min of pretreatment, disappeared almost entirely from holocellulose after 6 min of pretreatment. Select XG and xylan epitopes remained in the chlorite and $4 \mathrm{M} \mathrm{KOH} \mathrm{PC} \mathrm{extracts} \mathrm{of} \mathrm{all} \mathrm{pretreated} \mathrm{P.} \mathrm{trichocarpa} x$ P. deltoides 
samples, but only trace amounts of these epitopes remained in the 9 min and $12 \mathrm{~min}$ pretreated holocellulose samples. Taken together, these results suggest that delignified holocellulose containing few lignin-carbohydrate complexes experiences rapid hydrolysis of non-cellulosic polysaccaharides, particularly xylans and XGs.

Glycome profiles of birchwood xylan are presented in Figure 3 for only two pretreated samples ( 0 and $3 \mathrm{~min}$ ), because no solid material was recoverable after longer pretreatments. Because birchwood xylan is soluble in water at the concentration required for glycome profiling analysis ( $20 \mu \mathrm{g} / \mathrm{mL}$ water), no extraction of these materials was necessary prior to ELISA screening. Examination of the profile of the untreated birchwood xylan revealed that only xylan-5 through xylan-7 epitopes were present. Although xylan is linked to cellulose (Abramson et al., 2013; Faik, 2013), the influence of such interactions during pretreatment have not been considered previously. The absence of these interactions in birchwood xylan may have contributed to the rapid drop in epitope signal intensity observed in Figure 3 .

\subsection{Glucose release from Populus trichocarpa $x$ P. deltoides and model substrates}

The total glucose (monosaccharide and oligosaccharides) released from $P$. trichocarpa $x$ P. deltoides, holocellulose, and birchwood xylan was determined to be $0.016 \mathrm{~g}, 0.021 \mathrm{~g}$, and $0.014 \mathrm{~g}$, respectively. This result was expected as hydrothermal pretreatment has a limited impact on cellulose, the largest glucan source in the cell wall. Therefore, the balance of this study focused on xylan hydrolysis. 


\subsection{Xylooligomer production from Populus trichocarpa $x$ P. deltoides and model substrates}

The cumulative total xylose release (monosaccharide and oligosaccharides) from the three substrates is shown as a function of pretreatment time in Figure 4, and Figure 5 presents the release of straight chain xylooligomers according to degree of polymerization (DP) as a function of pretreatment time. The straight chain xylooligomers $(1 \leq \mathrm{DP} \leq 6)$ detected by UPLC-MS account for less than $15 \%$ of the total monosaccharides and oligosaccharides reported in Figure 4. The balance of oligosaccharides was likely longer straight chain oligomers as well as branched oligomers. Values in both figures are expressed as grams xylose equivalent per gram xylose equivalent in the untreated substrate (i.e., $P$. trichocarpa $x P$. deltoides, holocellulose, or birchwood xylan, as relevant).

Figure 4 shows that total xylose release from all substrates slowed significantly after approximately 9 min of pretreatment. When pretreatment of $P$. trichocarpa $x$ P. deltoides was increased to $192 \mathrm{~min}$ at $180^{\circ} \mathrm{C}$, total xylose production increased by only $9.5 \%$ relative to $12 \mathrm{~min}$ pretreatment. This deceleration in xylooligomer production is consistent with previous studies (Chen et al., 2010; Conner, 1984; Garrote et al., 1999; Kobayashi and Sakai, 1956; Lee et al., 1999; Maloney and Chapman, 1985; Zhu et al., 2012) that found a portion of xylan to be more resistant to hydrolysis.

Comparison of glycome profiles of $P$. trichocarpa $x P$. deltoides and holocellulose (Figures 1 and 2) suggested that reduction in lignin-carbohydrate complexes leads to rapid hydrolysis of polysaccharides, particularly xylans and XGs, 
as well as increased shortening of carbohydrates in pretreated solids. The total xylose release and xylooligomer DP distribution from P. trichocarpa $x$ P. deltoides and holocellulose further support this conclusion. Inspection of Figure 4 reveals that during the first 3 min of pretreatment, up to $96 \%$ more xylan was released from holocellulose than from $P$. trichocarpa $x$ P. deltoides. The release of xylan from $P$. trichocarpa $\mathrm{x}$ P. deltoides then increased by $300 \%$ after $3 \mathrm{~min}$. Comparison of Figures $5 \mathrm{a}$ and $5 \mathrm{~b}$ reveals that more xylose, xylobiose, xylotriose, and xylotetraose were produced from $P$. trichocarpa $x$ P. deltoides than from holocellulose after 3 min. This shift in xylooligomer distribution coincided with significant changes in the glycome profiles of $P$. trichocarpa $x$ P. deltoides (Figure 1). Compared to the 0 min pretreated sample, there was a significant decrease in the amount of material recovered in the chlorite and $4 \mathrm{M} \mathrm{KOH} \mathrm{PC} \mathrm{extractions} \mathrm{of} P$. trichocarpa $x P$. deltoides and in signal intensity of xylan-4 through xylan-7 antibody binding to these extracts of the 3 min pretreated sample, indicating a substantial decrease in ligninbound epitopes. This indirect observation of lignin removal was verified by compositional analysis of the solid residues: Figure 6 shows that $37.7 \%$ of lignin was released from $P$. trichocarpa $x P$. deltoides between 0 min and 3 min. A fraction of lignin was removed as phenolic monomers following depolymerization (Trajano et al., 2013) and another fraction may have been removed as colloidal material as the result of lignin-carbohydrate interactions (Hubbe et al., 2012, Trajano et al., 2013). No particulates were observed in the sampled hydrolysate, but any colloidal material would be too small for detection by the naked eye (Hubbe et al., 2012). A possible explanation for these observations of xylooligomer release is that intra-xylan bonds 
break more rapidly than lignin-carbohydrate bonds to generate small xylooligomers linked to lignin. The low solubility of these lignin-carbohydrate complexes would limit xylooligomer diffusion into the bulk liquid phase until sufficient lignin had been removed or until the oligomers were relatively small. Once this threshold was achieved, a sudden increase in the concentration of short-chain xylooligomers would be observed. Regardless of mechanism, lignin-carbohydrate interactions clearly impeded xylooligomer production at the outset of pretreatment.

Comparison of xylose release and xylooligomer DP distribution from holocellulose and birchwood xylan supports the theory that xylan-cellulose interactions may impede xylan hydrolysis. During the heating period, $43 \%$ more xylooligomers were released from birchwood xylan than from holocellulose (Figure 4). Although xylooligomer production from holocellulose exceeded xylooligomer production from birchwood xylan at the 3 min mark, $7.9 \%$ more xylooligomers were produced from birchwood xylan than from holocellulose after 12 min. In Figure 5c, it can be seen that negligible amounts of xylooligomers with $1 \leq \mathrm{DP} \leq 6$ were produced from birchwood xylan, whereas Figure $5 \mathrm{~b}$ shows that up to $38 \%$ of the total xylose released from holocellulose was straight chain xylooligomers with $1 \leq \mathrm{DP} \leq 6$. The parallels between these observations and those made for $P$. trichocarpa $\mathrm{x} P$. deltoides and holocellulose suggest that cellulose-xylan hydrogen bonds might have also limited xylooligomer release and diffusion. However, the differences between holocellulose and birchwood xylan were less striking. Therefore, the effects of xylan-cellulose hydrogen bonds on xylan hydrolysis appear to be smaller than those of lignin-carbohydrate interactions. 
Data on the solubility and diffusivity of poly- and oligosaccharides, lignin moieties, and lignin-carbohydrate complexes would be invaluable in verifying these hypotheses as well as assessing the effects of particle size on mass transfer; such data are currently unavailable. Song et al. (2012) found that total poly- and oligosaccharide yields from $0.75-1 \mathrm{~mm}$ particles were approximately $50-80 \%$ of the yields obtained using $<0.1 \mathrm{~mm}$ particles, which suggests that intraparticle mass transfer limitations may influence the results of this study.

Refining lignocellulosic biomass requires efficient and economical use of both cellulose and hemicellulose. Hemicellulose removal during pretreatment is a complex phenomenon, but hemicellulose has frequently been modelled as a homogeneous substrate, independent of the biomass structure. Biphasic models rely upon a lumped approach to describe some of the complexity, but do not describe the spatial distribution of hemicellulose within the cell wall or its interactions with lignin and cellulose. The results of this study highlight the inadequacy of this approach and the need to consider structure and polymer interactions when interpreting the results for pretreatment of $P$. trichocarpa $x P$. deltoides. It is very likely that similar considerations apply to other biomass species. Microscale models that incorporate mass transfer phenomena and spatial variations in hemicellulose could prove more mechanistically accurate, improve pretreatment strategies, and suggest new plant modifications for enhanced biomass utilization.

\section{Conclusions}

Glycome profiling of pretreated $P$. trichocarpa $x P$. deltoides, holocellulose, and birchwood xylan revealed cell wall loosening, differences in relative removal of 
glycan epitopes, and steady glycan epitope fragmentation and removal. Although the greatest total xylose production was from birchwood xylan followed by holocellulose, $P$. trichocarpa $\times$ P. deltoides produced the greatest amount of shortchained xylooligomers. Differences among the three substrates indicate that lignincarbohydrate bonds decrease hydrolysis rates, reduce carbohydrate shortening, and limit xylooligomers release, while xylan-cellulose hydrogen bonds appear to have a similar but smaller effect on hydrolysis. Thus, cell wall structure strongly affects pretreatment results.

\section{Supplementary Information}

The details of all monoclonal antibodies used are provided in SI Table 1. SI Figure 2 shows the cumulative total xylose release from Populus trichocarpa $x$ P. deltoides after 192 min flow-through hydrothermal pretreatment at $180^{\circ} \mathrm{C}$. SI Figure 3 shows the mass balances for the flow-through hydrothermal pretreatment of Populus trichocarpa $x$ P. deltoides, holocellulose, and birchwood xylan at $180^{\circ} \mathrm{C}$.

\section{Acknowledgements}

We thank the Office of Biological and Environmental Research in the DOE Office of Science for supporting this work through the BioEnergy Science Center (BESC). BESC is a U.S. Department of Energy Bioenergy Reseach Center supported by the Office of Biological and Environmental Research in the DOE Office of Science. This manuscript has been co-authored by a contractor of the U.S. Government under contract DE-AC05-00OR22725. We acknowledge support by the Ford Motor Company for the Chair in Environmental Engineering at the University of California Riverside that augments our ability to perform such 
research. HLT thanks the Natural Sciences and Engineering Research Council of Canada for a PGS-D scholarship. The generation of the CCRC series of plant cell wall glycan-directed monoclonal antibodies used in this work was supported by the NSF Plant Genome Program (DBI-0421683 and IOS-0923992). We also wish to thank Seokwon Jung, Georgia Institute of Technology, for preparing the holocellulose used in this study. No sponsor was involved in the study design; in the collection, analysis and interpretation of data; in the writing of the report; or in the decision to submit the article for publication.

\section{Competing interests}

CEW was a cofounder of Mascoma Corporation and until recently, Chief Development Officer and Chair of their Scientific Advisory Board. CEW was also a member of the Scientific Advisory Board of Mendel Biotechnology, Inc.

\section{References}

1. Abatzoglou, N.J., Chornet, E., Belkacemi, K., Overend, R.P., 1992. Phenomenological kinetics of complex systems: the development of a generalized severity parameter and its application to lignocellulosic fractionation. Chem. Eng. Sci. 47, 1109-1122.

2. Abramson, M., Shoseyov, O., Hirsch, S., Shani, Z., 2013. Genetic modifications of plant cell walls to increase biomass and bioethanol production, in: Lee, J.W. (Ed.), Advanced Biofuels and Bioproducts. Springer Science + Business Media, New York, pp. 315-338.

3. Ahlgren, P.A., Goring, D.A.I., 1971. Removal of wood components during chlorite delignification of black spruce. Can. J. Chem. 49, 1272-1275. 
4. Albersheim, P., Darvill, A., Roberts, K., Sederoff, R., Staehelin, A., 2011. Plant cell walls, Garland Science, New York.

5. Chen, X., Lawoko, M., van Heiningen, A., 2010. Kinetics and mechanism of autohydrolysis of hardwoods. Bioresource Technol. 101, 7812-7819.

6. Conner A.H., 1984. Kinetic modeling of hardwood prehydrolysis: Part I. Xylan removal by water prehydrolysis. Wood Fiber Sci. 16, 268-277.

7. DeMartini J.D., Pattathil, S., Avci, U., Szekalski, K., Mazumder, K., Hahn, M.G., Wyman, C.E., 2011. Application of monoclonal antibodies to investigate plant cell wall deconstruction for biofuels production. Energ. Environ. Sci. 4, 4332-4339.

8. Faik, A., 2013. Plant cell wall structure-pretreatment- The critical relationship in biomass conversion to fermentable sugars, in: Gu, T. (Ed.), Green Biomass Pretreatment for Biofuels Production. Springer, New York, pp. 1-30.

9. Garrote, G., Dominguez, H., Parajó, J.C., 1999. Mild autohydrolysis: an environmentally friendly technology for xylooligosaccharide production from wood. J. Chem. Technol. Biot. 74, 1101-1109.

10. Gray, M.C., Converse, A.O., Wyman, C.E., 2007. Solubilities of oligomer mixtures produced by the hydrolysis of xylans and corn stover in water at $180^{\circ} \mathrm{C}$. Ind. Eng. Chem. Res. 46, 2383-2391.

11. Hubbe, M.A., Sundberg, A., Mocchiutti, P., Ni, Y., Pelton, R., 2012. Dissolved and colloidal substances (DCS) and the charge demand of papermaking process waters and suspensions: a review. Bioresources. 7, 6109-6193.

12. Kim, Y., Kreke, T., Ladisch, M.R., 2013. Reaction mechanisms and kinetics of xylooligosaccharide hydrolysis by dicarboxylic acids. AICHE J. 59, 188-199. 
13. Kobayashi, T., Sakai, Y., 1956. Hydrolysis rate of pentosan of hardwood in dilute sulfuric acid. B. Agr. Chem. Soc. Japan. 20, 1-7.

14. Knox, J.P., 1997. The use of antibodies to study the architecture and developmental regulation of plant cell walls. Int. Rev. Cytol. 171, 79-120.

15. Kumar, R., Wyman, C.E., 2010. Key features of pretreated lignocelluloses biomass solids and their impact on hydrolysis, in: Waldon, K. (Ed.), Bioalcohol production :

Biochemical conversion of lignocellulosic biomass. Woodhead Publishing Ltd., Oxford, pp. 73-121.

16. Kumar, R., Hu, F., Hubbell, C.A., Ragauskas, A.J., Wyman, C.E., 2013. Comparison of laboratory delignification methods, their selectivity, and impacts on physiochemical characteristics of cellulosic biomass. Bioresource Technol. 130, 372-381.

17. Lee, Y.Y., Iyer, P., Torget, R.W., 1999. Dilute-acid hydrolysis of lignocellulosic biomass. Adv. Biochem. Eng. Biot. 65, 93-115.

18. Leu, S.Y., Zhu, J.Y., 2013. Substrate-Related Factors Affecting Enzymatic Saccharification of Lignocelluloses: Our Recent Understanding. Bioenerg. Res. 6, 405-415.

19. Liu, C., Wyman, C.E., 2003. The effect of flow rate of compressed hot water on xylan, lignin, and total mass removal from corn stover. Ind. Eng. Chem. Res. 42, 5409-5416.

20. Maloney, M.T., Chapman, T.W., Baker, A.J., 1985. Dilute acid hydrolysis of paper birch: kinetics studies of xylan and acetyl group hydrolysis. Biotechnol. Bioeng. 27, 355361.

21. Mok, W.S.L., Antal, M.J., 1992. Uncatalyzed solvolysis of whole biomass hemicellulose by hot compressed liquid water. Ind. Eng. Chem. Res. 31, 1157-1161. 
22. Pattathil, S., Avci, U., Baldwin, D., Swennes, A.G., McGill, J.A., Popper, Z., Bootten, T., Albert, A., Davis, R.H., Chennareddy, C., Dong, R., O'Shea, B., Rossi, R., Leoff, C., Freshour, G., Narra, R., O'Neill, M., York, W.S., Hahn, M.G., 2010. A comprehensive toolkit of plant cell wall glycan-directed monoclonal antibodies. Plant Physiol. 153, 514525 .

23. Pattathil, S., Avci, U., Miller, J.S., Hahn, M.G., 2012. Immunological approaches to plant cell wall and biomass characterization: Glycome profiling. Methods Mol. Bio. 908, $61-72$.

24. Saeman, J.F., 1945. Kinetics of wood saccharification: hydrolysis of cellulose and decomposition of sugars in dilute acid at high temperatures. Ind. Eng. Chem. 37, 43-52. 25. Sannigrahi, P., Ragauskas, A.J., Tuskan, G.A., 2010. Poplar as a feedstock for biofuels: A review of compositional characteristics. Biofuels, Bioprod. Biorefin. 4, 209226.

26. Sannigrahi, P., Kim, D.H., Jung, S., Ragauskas, A., 2011: Pseudo-lignin and pretreatment chemistry. Energy Environ. Sci. 4, 1306-1310.

27. Sluiter, A., Ruiz, R., Scarlata, C., Sluiter, J., Templeton, D., 2005. Determination of extractives in biomass. National Renewable Energy Laboratory, Golden.

28. Sluiter, A., Hames, B., Ruiz, R., Scarlata, C., Sluiter, J., Templeton, D., 2006.

Determination of sugars, byproducts, and degradation products in liquid fraction process samples laboratory analytical procedure. National Renewable Energy Laboratory, Golden. 29. Sluiter, A., Hames, B., Ruiz, R., Scarlata, C., Sluiter, J., Templeton, D., Crocker, D., 2011. Determination of structural carbohydrates and lignin in biomass laboratory analytical procedure. National Renewable Energy Laboratory, Golden. 
30. Song, T., Pranovich, A., Holmbom, B., 2012. Hot-water extraction of ground spruce wood of different particle size. Bioresources. 7, 4214-4225.

31. Trajano, H.L., Engle, N.L., Foston, M., Ragauskas, A.J., Tschaplinski, T.J., Wyman, C.E., 2013. The fate of lignin during hydrothermal pretreatment. Biotechnol. Biofuels. 6, 110.

32. Wickholm, K., Larsson, P.T., Iversen, T., 1998. Assignment of non-crystalline forms in cellulose I by CP/MAS C-13 NMR spectroscopy. Carbohydr. Res. 312, 123-129.

33. Willats, W.G.T., Steele-King, C.G., McCartney, L., Orfila, C., Marcus, S.E., Knox, J.P., 2000. Making and using antibody probes to study plant cell walls. Plant Physiol. Bioch. 38, 27-36.

34. Zhang, D.C., Pu, Y.Q., Chai, X.S., Naithani, V., Jameel, H., Ragauskas, A.J., 2006. Elucidating carboxylic acid profiles for extended oxygen delignification of high-kappa softwood kraft pulps. Holzforschung. 60, 123-129.

35. Zhu, W., Houtman, C.J., Zhu, J.Y., Gleisner, R., Chen, K.F., 2012. Quantitative predictions of bioconversion of aspen by dilute acid and SPORL pretreatments using a unified combined hydrolysis factor (CHF). Process Biochem. 47, 785-791.

\section{Figure Captions}

Fig. 1. Glycome profile of untreated and pretreated Populus trichocarpa x P. deltoides. The residual solids recovered after each pretreatment were extracted with solvents in the following order of increasing severity: $50 \mathrm{mM}$ ammonium oxalate, $50 \mathrm{mM}$ sodium carbonate, $1 \mathrm{M} \mathrm{KOH}, 4 \mathrm{M} \mathrm{KOH}$, sodium chlorite, and a second $4 \mathrm{M} \mathrm{KOH}$ extraction (4M KOH PC). Extracts were loaded onto ELISA plates at a constant carbohydrate 
loading and screened against an array of plant glycan-directed monoclonal antibodies (right legend panel). Each column represents an increase in pretreatment time and within each column is the series of sequential extracts. Antibody binding is represented as colored heat maps; black signifies no binding, pink/red represent intermediate binding, and bright yellow represents the strongest binding. The bar graphs at the top indicate the amount of soluble material recovered in each extract per gram of alcohol insoluble residue (AIR).

Fig. 2. Glycome profile of untreated and pretreated holocellulose isolated from Populus trichocarpa $x$ P. deltoides. See Figure 1 caption for legend details.

Fig. 3. Glycome profile of untreated and pretreated birchwood xylan. See Figure 1 caption for legend details.

Fig. 4. Cumulative total xylose (monosaccharide and oligosaccharides) released from Populus trichocarpa $x$ P. deltoides (squares), holocellulose (circles), and birchwood xylan (x) during flow-through pretreatment in grams xylose equivalent per gram of xylose in the untreated substrate as a function of pretreatment time. Lines are added to assist with visualization.

Fig. 5. Cumulative release of straight chain xylooligomers by degree of polymerization (DP) during flow-through pretreatment of (a) Populus trichocarpa $x$ P. deltoides (PtPd) , (b) holocellulose (HC), and (c) birchwood xylan (BX) in grams xylose equivalent per gram of xylose in the untreated substrate as a function of pretreatment time. Xylose (DP1), xylobiose (DP2), xylotriose (DP3), xylotetraose (DP4), xylopentaose (DP5), and xylohexaose (DP6) were measured. Lines are added to assist with visualization. 
Fig. 6. Cumulative removal of Klason lignin during flow-through hydrothermal pretreatment of Populus trichocarpa $x$ P. deltoides at selected times. The lignin removal was calculated by subtracting the mass of lignin measured in solids left in the reactor at each time from the mass of lignin in the biomass initially loaded into the reactor. Line is added to assist with visualization. 


\section{Tables and Figures}

Table 1. Composition (wt\%) of Populus trichocarpa $x$ P. deltoides, holocellulose isolated from P. trichocarpa $x$ P. deltoides and birchwood xylan (Sigma-Aldrich, Lot 010M0169). Standard deviations of triplicate measurements are provided in brackets.

\begin{tabular}{cccc}
\hline $\begin{array}{c}\text { Substrate } \\
\text { Glucan }\end{array}$ & $\begin{array}{c}\text { Xylan } \\
(\mathrm{wt} \%)\end{array}$ & $\begin{array}{c}\text { Klason lignin } \\
(\mathrm{wt} \%)\end{array}$ \\
\hline $\begin{array}{c}\text { P. trichocarpa } \mathrm{xt}) \\
\text { Holocellulose }\end{array}$ & $50.5(0.2)$ & $11.5(0.4)$ & $22.7(0.1)$ \\
Birchwood xylan & $1.6(0.9)$ & $21.2(0.2)$ & not detected \\
\hline
\end{tabular}


Table 2. Concentration profiles of acetonitrile with $10 \mu \mathrm{M}$ sodium acetate (Solvent A) and water with $10 \mu \mathrm{M}$ sodium acetate (Solvent B) during ultra-high performance liquid chromatography-mass spectrometry (UPLC-MS) analysis of pretreatment hydrolysate.

\begin{tabular}{cccc}
\hline $\begin{array}{c}\text { Time } \\
(\mathrm{min})\end{array}$ & $\begin{array}{c}\text { Concentration of } \\
\text { Solvent A (v/v\%) }\end{array}$ & $\begin{array}{c}\text { Concentration of } \\
\text { Solvent B (v/v\%) }\end{array}$ & $\begin{array}{c}\text { Flow rate } \\
(\mathrm{mL} / \mathrm{min})\end{array}$ \\
\hline 0 & 80 & 20 & 0.5 \\
1.0 & 80 & 20 & 0.5 \\
2.0 & 70 & 30 & 0.5 \\
2.1 & 5 & 95 & 0.5 \\
3.0 & 5 & 95 & 0.5 \\
3.1 & 80 & 20 & 0.5 \\
\hline
\end{tabular}




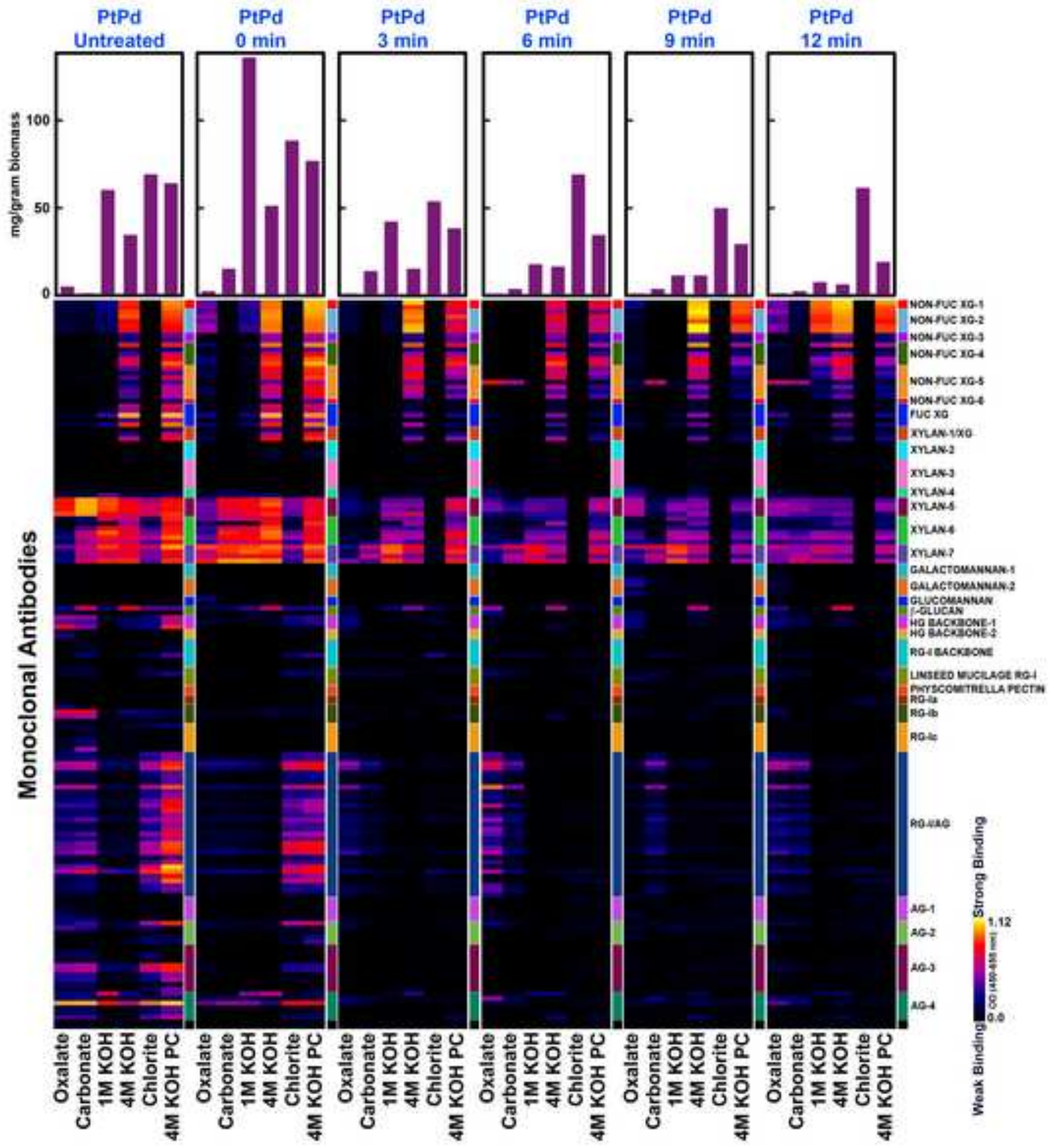




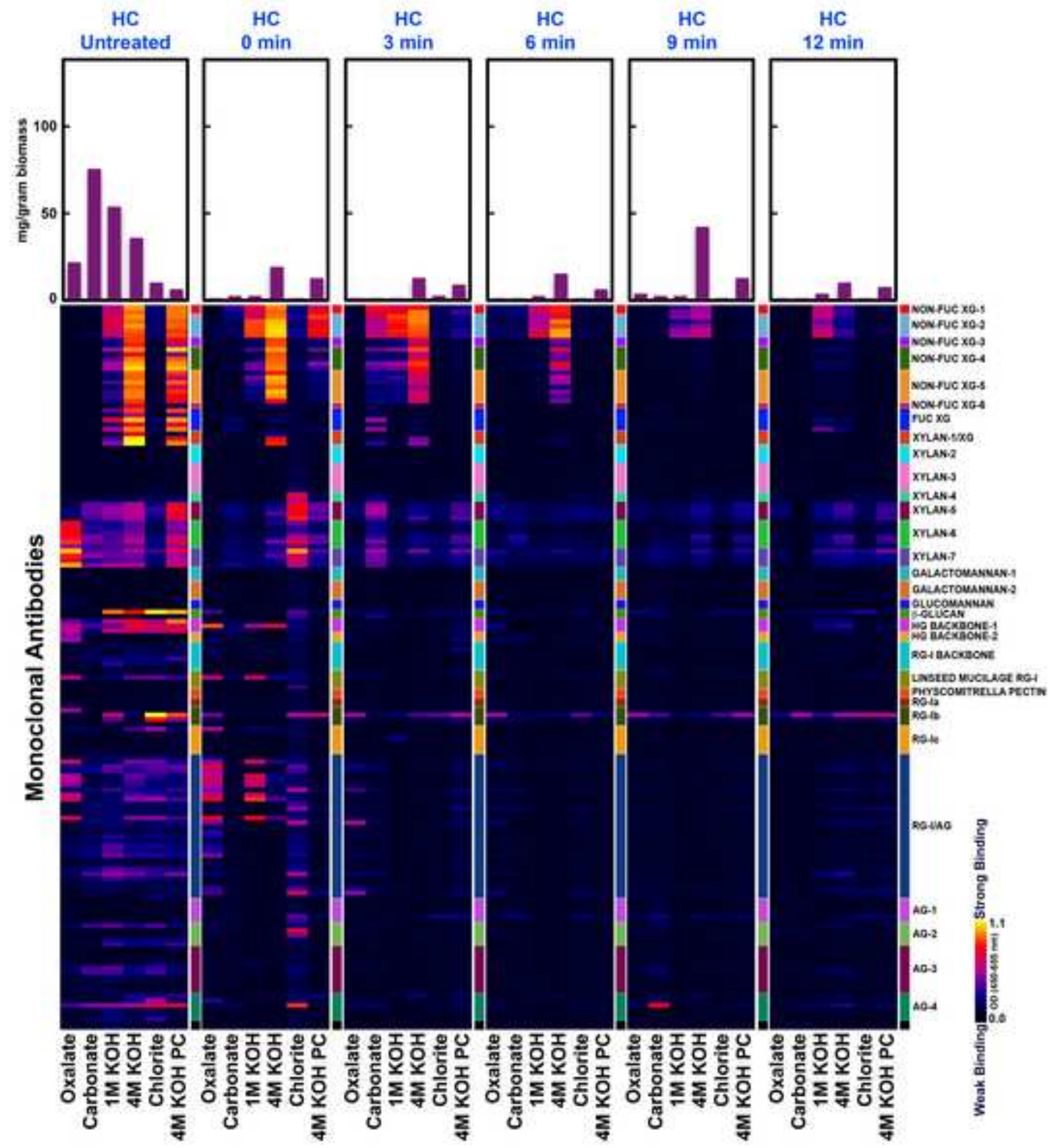




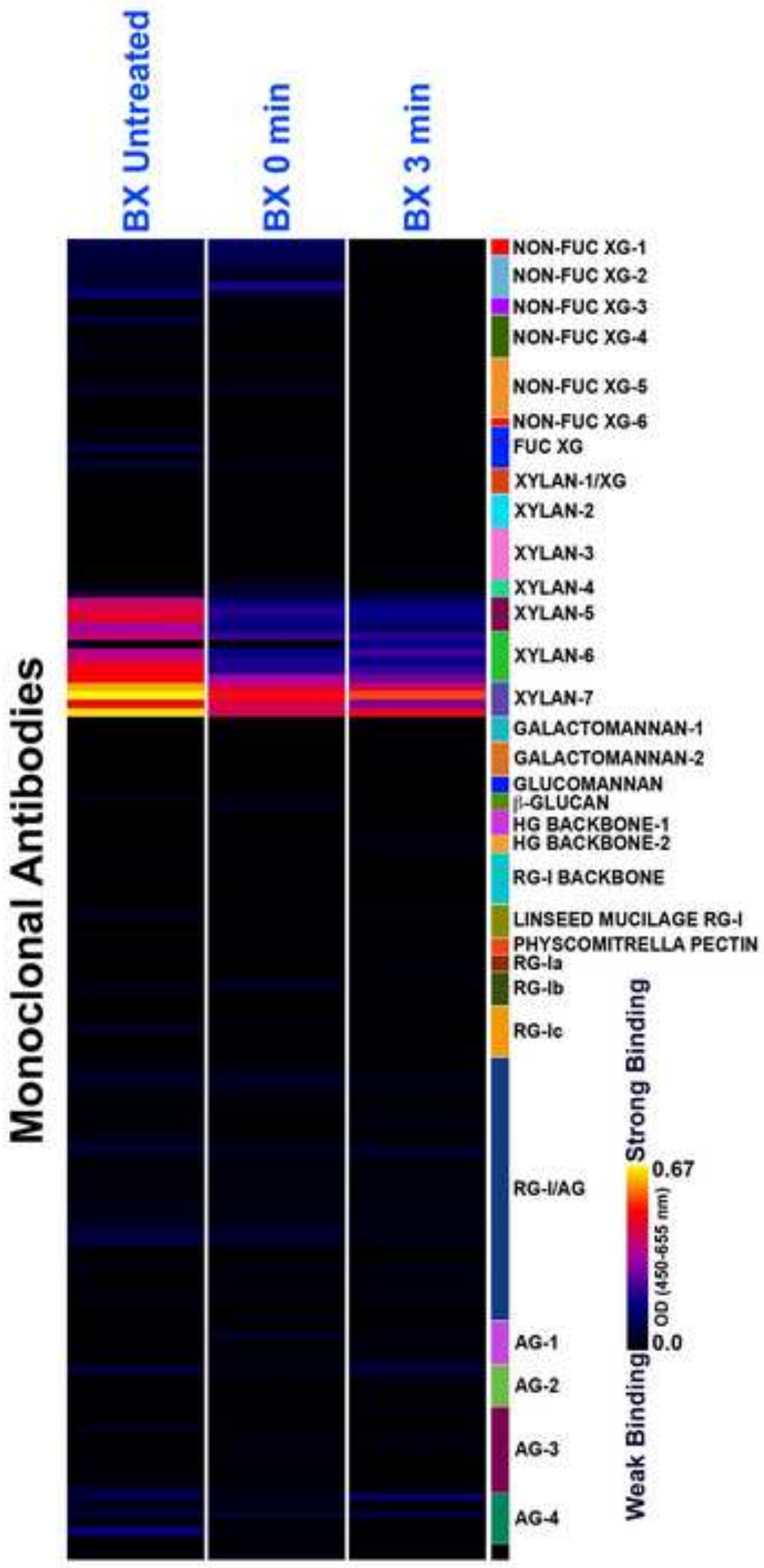




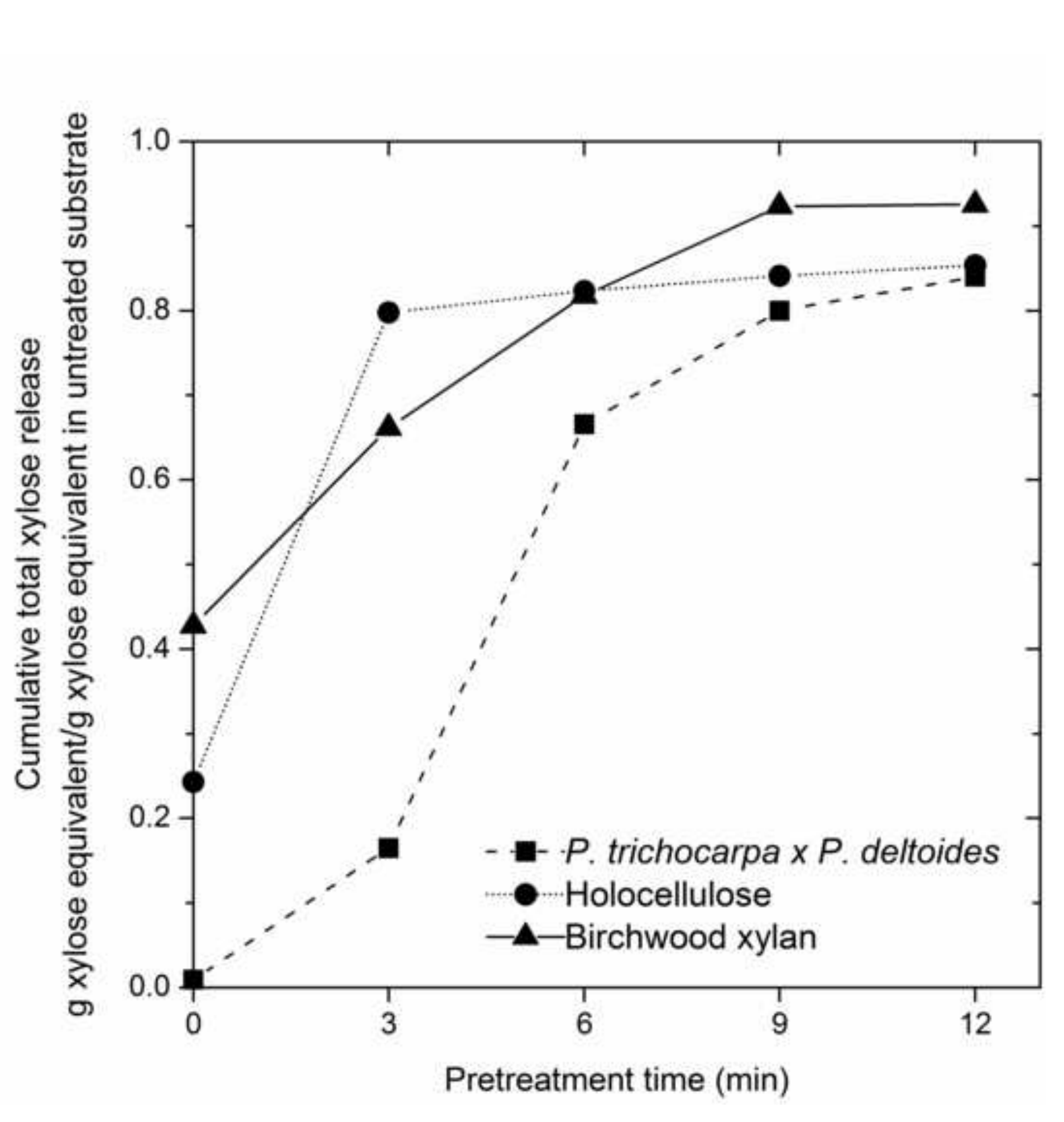

Figure 4

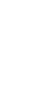
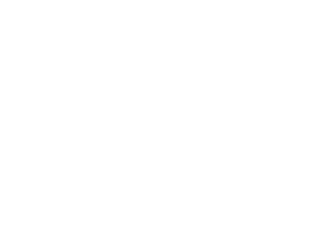

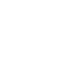
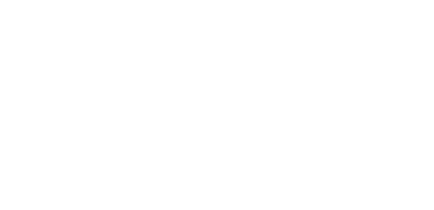


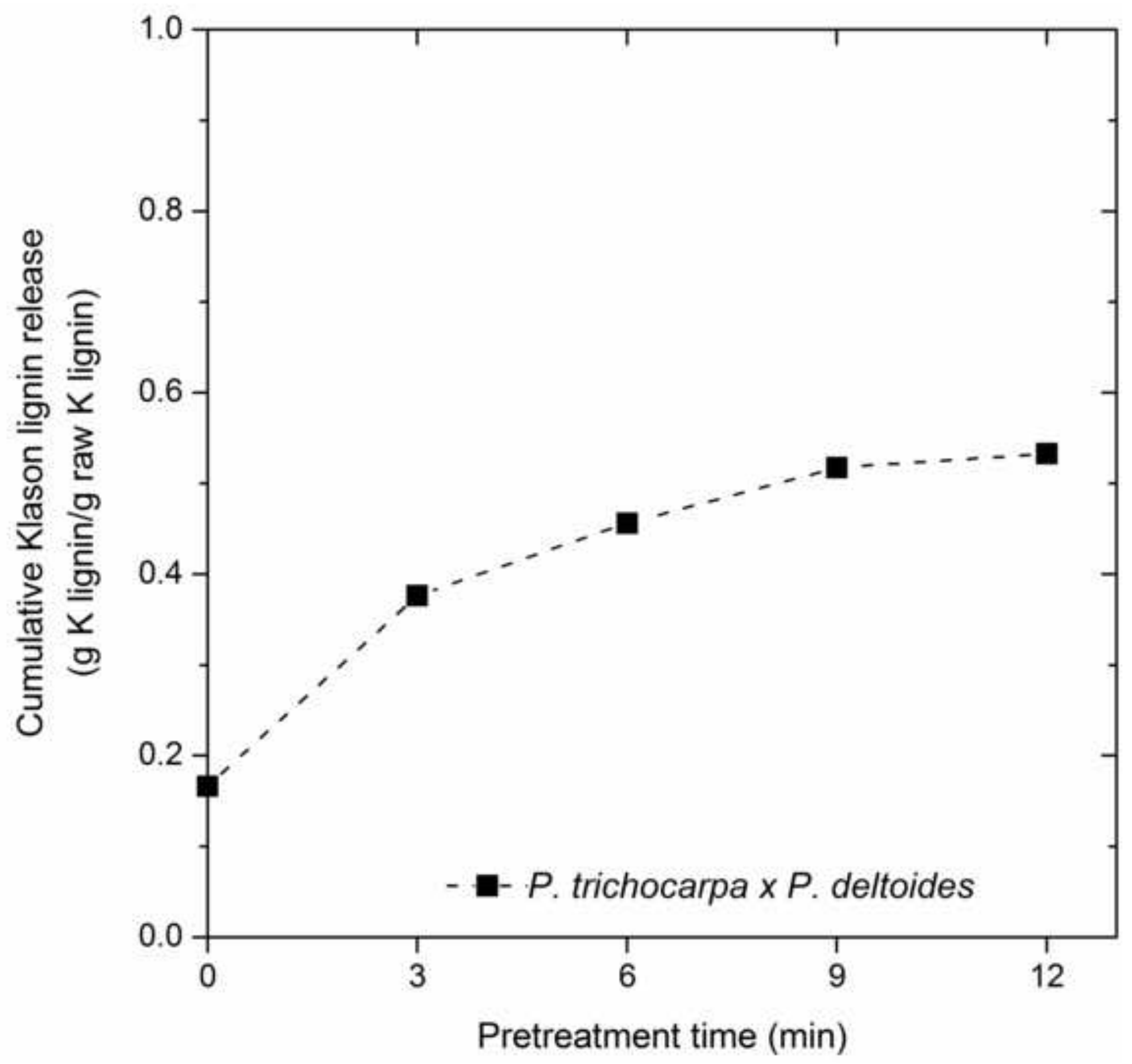

